\section{The facilitation of picture discrimination after object discrimination learning in the neonatal monkey and probably vice versa*}

\author{
ROBERT R. ZMMMERMANN, University of Montana, Missoula, Mont. 59801 \\ and \\ JULIAN HOCHBERG, Columbia University, New York, N.Y. 10037
}

Two groups of 20-day-old rhesus monkeys were trained to discriminate a three-dimensional vs a two-dimensional object or a pictorial representation of the objects. Object discrimination was significantly faster than pictorial discrimination, and reversal transfer tests showed significant savings in both cases. However, Ss trained on the object-picture sequence completed all training before any Ss completed the initial picture problem. The performance of the picture-object group in transfer tests did not differ significantly from other groups of comparable age.
It has recently been found that infant monkeys are capable of transferring a learned object discrimination to a picture or pictorial representation of these objects (Zimmermann \& Hochberg, 1970). These studies demonstrated that lines discriminable stimuli for the infant monkey, and, more important, when these lines correspond to the edges of dihedral angles of solid objects, the monkeys respond to the edges as they do to the lines. In other words, there was an equivalence of outline shapes and objects' edges that is also characteristic of human shape perception (Hochberg \& Brooks, 1962).

This preliminary research had difficult to evaluate the source of the equivalence of lines and edges as the differential cue, and animals varied considerably in age. One monkey less than 1 month of age at the time of transfer from objects to pictures showed significant savings; older animals had higher transfer scores. Furthermore, older animals with extensive discrimination learning experience had higher transfer scores to more subtle outline drawings than did less experienced or younger monkeys. Finally, repeated testing on a variety of picture-like forms improved transfer performance.

The present experiment was original learning of object discrimination and discriminations of pictorial representations of objects in naive neonatal rhesus macaques and to test for subsequent transfer to pictures and objects.

*This research was supported in part by Grant No. NSF-G 9601 to J. Hochberg from the National Science Foundation and by Grant No. M-4516 to R. Zimmermann from the National Institutes of Health. on a plane surface could function as several shortcomings which made it undertaken to compare the rate of
TEST PROCEDURES

All of the Ss began pretraining in a Grice-type discrimination apparatus (Zimmermann, 1961) on the first day of life and continued until 20 days of age, when formal discrimination learning problems were introduced. Discrimination learning and transfer tests were made in a discrimination maze, 24 in. long, 18 in. wide, and 12 in. high. A starting box, 6 in. square, was separated from a choice chamber by a vertically sliding Plexiglas door. The choice chamber was. 8 in. long and was separated from the goal chambers by an opaque vertically sliding door. Both goal chambers were 8 in. wide and 10 in. deep and were separated by an opaque partition. All stimuli were mounted on boards which were inserted in the rear of the goal chamber, and a hole was drilled in the center of each stimulus to permit the nipple of a nursing bottle to be inserted.

Briefly, the procedures were as follows: The $S$ was placed in the startbox, and the transparent door separated it from the choice chamber. The opaque door separated the $S$ visually from the discriminanda inserted in the rear of the divided goal area. When the opaque door was raised, the $S$ was delayed in the startbox for approximately 2 sec. The transparent door was then raised, and the $S$ was permitted to approach the rear of the maze.

The Ss were trained to locomote from the startbox to one of the goal chambers before discrimination training was begun. To avoid the development of position habits, the Ss were forced to go to each of the only in the testing situation described below.

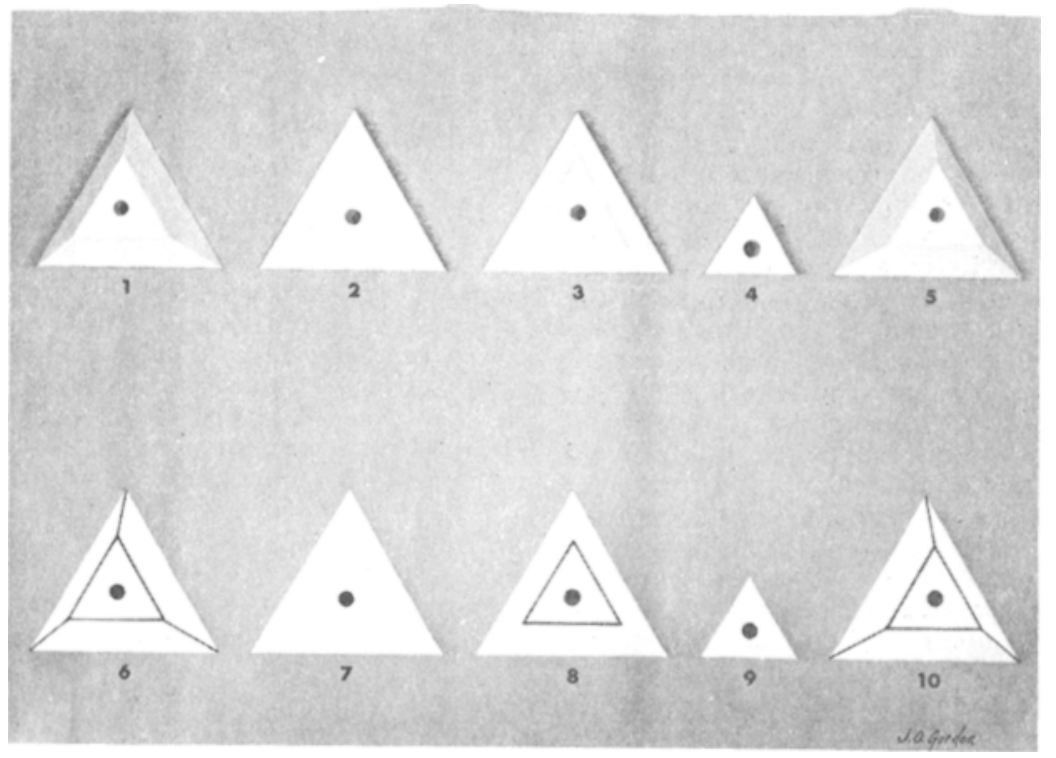

Fig. 1. Artist's impression of object and pictorial stimuli. 


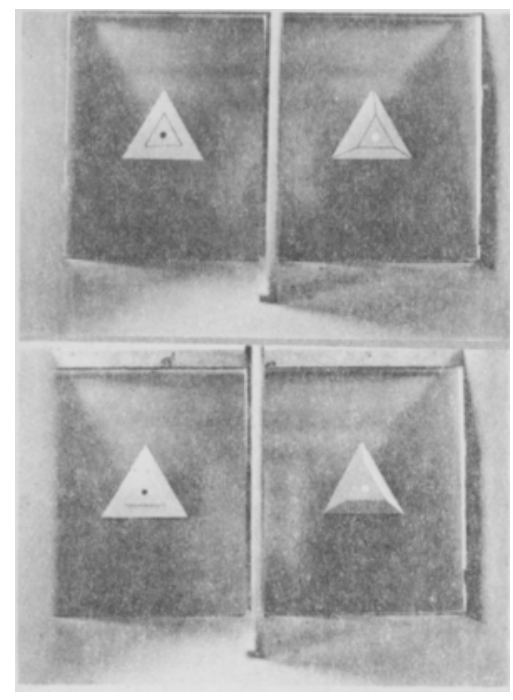

Fig. 2. Representative objects and pictures in position in discrimination maze.

chambers an equal number of times. This was done by blocking one of the goal chambers with an opaque door. Each $S$ was reinforced in each goal chamber an equal number of times. Training and testing was conducted five times a day at 2 -h intervals, five trials per session, beginning at 8:00 a.m. As reinforcement, the infants were permitted to drink approximately 5-10 cc of standard monkey milk formula per correct trial. On incorrect trials during testing, the Ss were detained in the nonrewarded goal chamber for approximately 15 sec. At 30 days of age, all Ss were given fruit and monkey chow during the day. Water was available in the living cage at all times.

Two groups of four infant monkeys each began discrimination learning at 20 days of age. An artist's impression of the stimuli used in the experiment is shown in Fig. 1. Numbers 1-5 represent the objects, and Nos. 6-10 represent the pictorial representations of the figures. One group was trained to discriminate between a truncated pyramid (Nos. 1 and 5), 3 in. each side at the base and $1 \frac{11 / 2}{\mathrm{in}}$. at the apex, and three triangular patterns cut from 1/8-in. Masonite representing the apex (No. 4), the base (No. 2), and the apex mounted on the base (No. 3) of the pyramid. A second group of four monkeys was trained to discriminate perspective drawings of the stimuli described above (Nos. 6-10) derived from photographs taken from the choice point of the maze. The stimuli were painted white and mounted on gray Masonite boards that fitted into the rear of the maze. A representative pair of stimuli is shown in position in the apparatus in Fig. 2. Only one of the triangles was paired with the pyramids at a single five-trial session. It required 3 days of testing before all of the triangles could be presented an equal number of times in the prescribed test periods, and, within that time, each triangle would have appeared 25 times. Two Ss were rewarded for approaching the pyramid and pyramid drawing and the remaining two for approaching the triangles. The criterion of learning was 21 out of 25 correct responses to any two of the three pairs for 2 successive days, and a noncorrection procedure was used throughout.

After achieving the criterion of learning, the Ss received transfer of training tests to a reverse set of dimensions. For example, an $S$ rewarded at the truncated pyramid (object) was now rewarded at the pictorial triangles, while an $\mathrm{S}$ rewarded at the pictorial triangles was rewarded at the truncated pyramid. Both groups were counterbalanced in this respect. RESULTS

The rate of learning to the three different triangles did not differ significantly in either group, nor was there any difference between three-dimensional positive as opposed to two-dimensional positive. The original learning and reversal curves are shown in Fig. 3. For the construction of the group curves, the criterion score was inserted after an individual had achieved the criterion and began the transfer tests.

Acquisition of the discrimination between objects was considerably more rapid than acquisition of the picture discrimination. Ss tested on the object discrimination required 11.25 days on the average to reach the criterion (not counting the 6 criterion days), while the Ss trained on picture discrimination required 43.50 days on the average to achieve the same level of performance. There was no overlap in the distribution of criterion training days between the two groups. In the transfer of training tests, both groups showed a negative transfer effect when the rewarded dimension was reversed and object or pictures substituted. Only the group that transferred from pictures to objects dropped significantly below chance in the early transfer trials. This negative transfer effect was quickly overcome by this group, and the criterion of learning was achieved in 2.25 days of training. The object-to-picture group fell slightly below chance in the initial trials and required 13.5 days to achieve the same level of performance. Again, the distribution of criterion scores for the two groups did not overlap.

The most surprising result is the fact that the rate of learning to the different triangles did not differ. These stimuli were deliberately selected to produce an ordered difference. That is, the apex triangle provided the largest difference from the pyramid, and the apex mounted on the base provided the least difference, with the base triangle falling in the middle (at least as it appeared to the human eye from a monkey's eyeview of the situation). Indeed, the selection of the criterion was based on the assumption that the

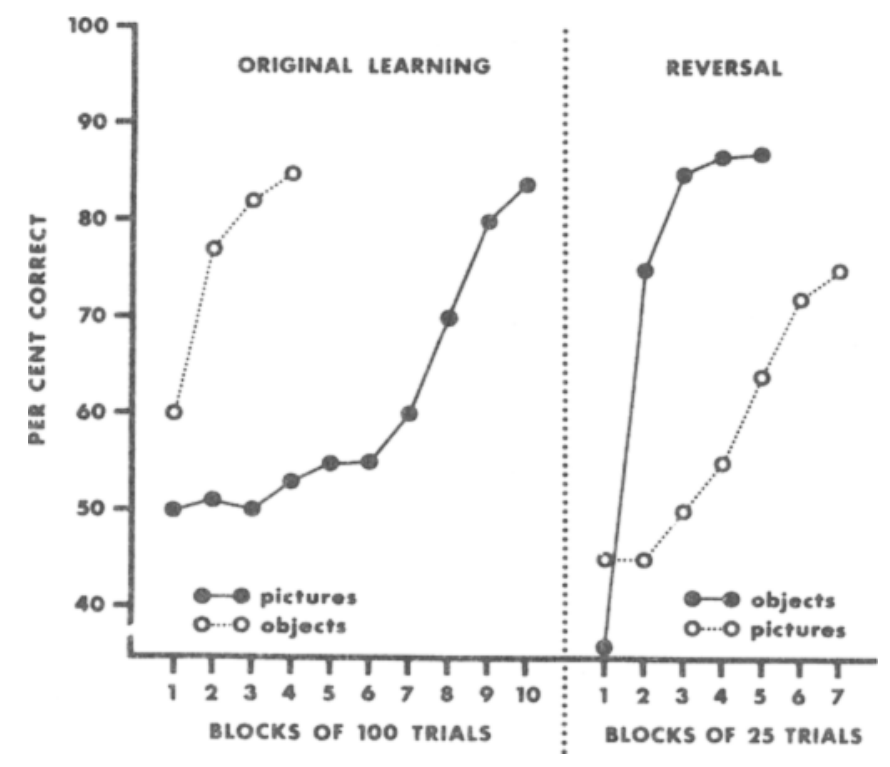

Fig. 3. Original learning and reversal to objects and pictures. In the reversal condition the $S$ was shifted from objects to pictures or vice versa, and the opposite dimension (three dimensional or two dimensional) from original learning was rewarded. 
infants would learn to discriminate the base and the apex triangles from the pyramid much more rapidly than the apex mounted on the base.

If the experimental design were treated as a simple 2 by 2 Latin square it is obvious that significant savings result in both groups (43.5 to 13.5 in the picture comparisons and 11.25 to 2.25 in the object comparisons). Age, of course, is a confounding factor, but in the case of the object-to-picture group, at least a portion of the savings is independent of age, since these Ss were 50.75 days of age on the average when they achieved the criterion of learning to pictures, while the picture-to-object group did not reach this level of performance on their first picture problem until 63.5 days of age. In the picture-to-object group, any savings are hopelessly confounded with age, since animals of this age with any past history of discrimination learning require approximately 25 trials to learn a simple object discrimination. However, the performance here is consistent with the finding that it takes animals of this age 50 to 75 trials to learn a simple reversal problem (Zimmermann \& Torrey, 1965). In spite of the age confounding, it is apparent that the animals in the picture-to-object group were responding to the edges of the objects as if they were equivalent to the lines on a plane, since they showed significant negative transfer in the early trials.

The difference in rate of learning to objects vs pictures is consistent with the long history of the superiority of stereometric discriminations over planimetric discriminations in the monkey (Meyer et al, 1965). The savings and rate of reversal learning found in the young monkeys may readily be interpreted as reflecting the acquired distinctiveness of the cues in this experiment. The primary cue that differentiated the triangles from the pyramid, both in objects and in drawings, was the dihedrals that are present in the object and drawing of the pyramid.

The experiment shows that object discrimination learning facilitates the discrimination of pictures of that object in the infant monkey and that this improved performance is not solely a function of normal biological maturation or some incidental learning process that is the result of living in a normally lighted visual world.

REFERENCES

HOCHBERG, J., \& BROOKS, V. Pictorial recognition as an unlearned ability: $A$ study of one child's performance. American Journal of Psychology, 1962, 75, 624-628.

MEYER, D. R.. TREICHLER, F. F., \& MEYER, P. M. Discrete trial training techniques and stimulus variables. In $A$. M. Schrier, H. F. Harlow, and F. Stollnitz (Eds.), Behavior of nonhuman primates: Modern research trends. Vol. 1. New York: Academic Press, 1965. Pp. 1-50.

ZIMMERMANN, R. R. Analysis of discrimination learning capacities in the infant thesus monkey. Journal of Comparative \& Physiological Psychology, $1961,54,1-10$.

ZIMMERMANN, R. R.. \& HOCHBERG, H Responses of infant monkeys to pictorial representations of a learned visual discrimination. Psychonomic Science, 1970, 18, 307-308.

ZIMMERMANN, R. R., \& TORREY, C. C. Ontogeny of learning. In A. M. Schrier, H. F. Harlow, and $F$. Stollnitz (Eds.), Behavior of nonhuman primates: Modern research trends, Vol. II. New York: Academic Press, 1965. Pp. 405-448.

\section{CURRENT LITERATURE ON DISCRIMINATION LEARNING IN ANIMALS}

ASATO, H. (Veterans Administration Hospital, Phoenix, Ariz. 85012), \& FLEMING, D. E. The relationship between EEG amplitude and the rate of classical conditioning. Psychonomic Science, 1971, 22, 37-38.

BRACKBILL, R. M. (Franklin and Marshall College, Lancaster, $\mathbf{P a}$. 17604), \& BROOKSHIRE, K. H. Conditioned taste aversions as a function of the number of CS-US pairs. Psychonomic Science, 1971, 22, 25-26.

BRUNING, J. L. (Ohio University, Athens, Ohio 45701), SCHMECK, R. R., \& SILVER, A. I. Frustration, competing responses, and error making. Psychonomic Science, $1971,22,47-48$.

DAVENPORT, D. G. (St. Louis University, St. Louis, Mo. 63103), OLSON, R. D., \& OLSON, G. A. Preliminary analysis of partial reinforcement in discriminated avoidance. Psychonomic Science, 1971, 22, 9-11.

DUTCH, J. (Victoria University of Wellington, Wellington, New Zealand), \& BROWN, L. B. Approach behavior to visual stimuli in chicks and rats. Psychonomic Science, 1971, 22, 49-50.

GILBERT, R. M. (Alcoholism and
Drug Addiction Research Foundation, Toronto 179 , Ontario, Canada). Signal functions in discriminated avoidance behavior. Journal of the Experimental Analysis of Behavior, 1971, 15, 97-108

GROESBECK, R. W. (Gonzaga University, Spokane, Wash. 99202), \& DUERFELDT, P. H. Some relevant variables in observational learning of the rat. Psychonomic Science, 1971, 22, 41-43.

GROSSEN, N. E. (Sacramento State College, Sacramento, Calif. 95819). Effect of aversive discriminative stimuli on appetitive behavior. Journal of Experimental Psychology, 1971, 88, 90-94.

KINSBOURNE, M. (Division of Pediatric Neurology, Duke Medical Center, Durham, N.C. 27706). Discrimination of orientation by rats. Psychonomic Science, 1971, 22,50 .

LEVINSON, D. M. (V.A. Hospital, Kansas City, Mo. 64128), HOTTMAN, T. J., \& SHERIDAN, C. L. Assessment of the generality of enhanced learning following unilateral lesions of posterior neocortex in rats. Psychonomic Science, 1971, 22, 1-3.

MANKINS, S. L. (Bowling Green State
University, Bowling Green, Ohio 43402), \& RAGUSA, D. M. Effects of interpolated escape training on discriminated leverpress avoidance. Psychonomic Science, 1971, 22, 15-16.

MILLER, L. (Western Washington State College, Bellingham, Wash. 98225), \& PRICE, R. D. Stimulus compounding and response summation with an instrumental running response. Psychonomic Science, 1971, 22, 52-53.

OLSON, R. D., DAVENPORT, D. G. (St. Louis University, St. Louis, Mo. 63103), \& KAMICHOFF, N. C. Discriminated avoidance and the partial reinforcement effect. Psychonomic Science, 1971, 22, 12-14.

POWELL, R. W. (Department of Behavioral Science, University of South Florida, Tampa, Fla. 33620). Some effects of punishment shock intensity upon discriminative responding. Journal of the Experimental Analysis of Behavior, 1971, 15, 109-116.

WINTON, A. S. W. (University of Auckland, Auckland, New Zealand), \& BEALE, I. L. Peak shift in concurrent schedules. Journal of the Experimental Analysis of Behavior, $1971,15,73-81$. 